\title{
Influence of Demographic Factors on the Psychometric Properties of School Administrators Leadership Scale
}

\author{
Catherine Nkiruka Elechi \\ Department of Educational Foundations, \\ Enugu State University of Science and Technology, Enugu, Enugu State
}

\begin{abstract}
Although the issue of leadership has been widely emphasized in school administration, the aspect that pertains to its measurement has not gained prominence in current research discourse. It must also be appreciated that the validity of most research findings in school administrative leadership have been inherently questionable owing to the paucity of a standard measuring instrument in the leadership skill domain. Considering also the current inclusion of leadership skill as basis for appointment of school administrators, one may wonder how this objective could be realized without a valid and reliable measuring instrument. Also, because these principals are of varying characteristics in terms of gender and job experience, it may be argued that the responses of school administrators on leadership skill assessment scales may be influenced or even dependent on these variables. While school administrative leadership scales are used holistically irrespective of demographic issues, researchers in the field of instrumentation have lost focus of the implication(s) of demographic factors on psychometric attributes of such scales. Incidentally the psychometric properties of such administrative leadership scales are to a large extent influenced by demographic variables such as gender, location and job experience. In a nutshell, this study, using an instrumentation design, revealed the differential factor matrix, internal consistency indices and stability of a school administrative leadership scale across demographic variables. In view of the current emphasis on effective leadership and the acquisition of good leadership skill, it has become obviously necessary that demographic factors be considered and built into the leadership skills assessment templates of prospective school administrators.
\end{abstract}

\section{Introduction}

One of the concerns of the changing global economy is leadership. In today's global society, organizations have to deal with challenges as well as increasingly volatile competition. Therefore leaders' effectiveness has become a very important issue that must be explored in order to understand how to motivate employees and help them to achieve organizational goals.

Leadership has been described as a process of social influence in which one person can enlist the aid and support of others in the accomplishment of a common task. According to Adesina in Elechi, it is the quality, which in theory signifies the ability of a person or group of people to persuade others to act by inspiring them and making them believe in a proposed course of action [2]. It implies followership which exists within an organizational context.

Leadership has been viewed from a number of theoretical and empirical perspectives. Theoretical approaches, to leadership include behavioral approach, the least preferred Coworker (LPC) theory, the pathgoal model, Vroom-Yetton-Jago model and other few contemporary approaches such as leader-member exchange model and the Hersey and Blanchard model. Like other key organizational behavior terms, such as personality, leadership is used in a variety of ways. According to Moorhead and Griffin, leadership is both a process and a property [7]. As a process, leadership involves the use of non-coercive influence. As a property, it is the set of characteristics attributed to someone who is perceived to use influence successfully. This implies that leadership is a quality, which in theory signifies the ability of a person or a group of people to persuade others to act by inspiring them and making them believe that a proposed course of action is the correct one. Going by these conceptualizations, leadership involves neither force nor coercion. An administrator who relies on force and formal authority to direct the behavior of subordinates is not practicing leadership [1].

Mcshane and VonGlinow identified two dimensions of leadership; initiating structure and consideration [5]. According to McShane and VonGlinow initiating structure refers to the leaders' behavior in delineating the relationship between himself and members of the work group, and endeavoring to establish well defined patterns of organization, channels of communication and methods of procedure [5]. On the other hand, consideration implies the behavior indicative of friendship, mutual trust, respect and warmth in the 
relationship between the leader and members of his staff.

Leadership can be perceived as a particularly emotion-laden process, with emotions entwined with the social influence process. In an organization like a school, the leader's mood has some effects on his/her group. Studies on leadership have shown various leadership styles exhibited by leaders especially school principals. Different situations call for different leadership styles and these styles can also be influenced by certain demographic factors such as age, gender, social class, professional experience, level of education, family or race/ethnicity. A demographic factor like gender covaries with leadership style. When men and women come together in groups, they tend to adopt different leadership styles. This goes in line with Vroom in his theories of leadership which provided a model that depicts pronounced differences in leader skills of individuals arising from gender differences [10]. He went ahead to trace these differences to hormonal effects. However, it is important to note that these sex differences are only tendencies and do not manifest themselves within men and women across all groups and situations.

In view of the fact that one of the concerns of the changing global economy is leadership, and due to the fact that education is the instrument for national transformation and technological development, there is a need for more emphasis to be laid on effective teaching and learning in schools. Effective teaching and learning is a function of leadership. To achieve the goals of education at all levels requires effective leaders.

In the past years especially with the creation of Enugu State, Nigeria, which catalyzed hurried appointment of school principals, the status of secondary education has begun to change [2]. The degree of teacher redundancy, overt truancy, disruptive behaviors on the part of students and the associated poor academic achievement go a long way to confirm the degenerating status of secondary education. Although the current trends in secondary school management have been speculated to stem from leadership styles of principals, such assumptions lack empirical backings. Although studies on leadership appear to be quite copious, it must be appreciated that the focus of researchers has not been on instrumentation in leadership traits. It must also be appreciated that the validity of most research findings in school administrative leadership have been inherently questionable. This is because of the non-availability of a standard measuring instrument in that leadership skill domain.

In view of the current emphasis on effective leadership and the acquisition of good leadership skill as a requisite for appointment and promotion of principals in Enugu State, Nigeria, it has become obviously necessary that a valid, and reliable school administrative leadership scale that is stable across certain demographic traits be developed for researchers in the field of leadership and also for effective screening of "would be" and already appointed principals [8].

\subsection{Statement of the Problem}

The current high rate of administrative lapses in secondary schools, are sources of distress to the education ministry and the entire public where investments on education need be justified. Although the general consensus about these problems is that secondary schools lack good leaders who could coordinate both the human and material resources of the schools towards the realization of set goals, it must be appreciated that the extent of acquisition of leadership skills among school principals is merely speculated [2]. This is true because, most studies on leadership were conducted without a valid and reliable measuring tool in that specific skill domain. According to Asike in (Elechi), most researchers in the field of leadership had gone ahead to collect data on leader skills and behavior using ordinarily face-validated tools [2]. For the fact that the psychometric attributes of the instruments were not ascertained, it is quite obvious that whatever data collected with such an instrument and conclusions derived from them are also questionable. While also much emphasis is given to research in leadership and productivity, it will be very necessary if researchers in the field are equipped with valid, reliable and stable instruments.

Considering also the fact that leadership is a personality trait, whatever instrument designed to measure it must be stable across demographic variables such as gender, job experience, age etc. It therefore becomes necessary that in developing an instrument of this type, its stability across some demographic factors need be explored. In fact research issues at this point are not only developing a valid and reliable scale but also the extent to which the instrument is stable across major demographic variables. This study is therefore faced with the problem of exploring the psychometric properties of a school administrative leadership scale.

Specifically the study explored the influence of gender of the principals on the school administrative leadership scale (SALS)

\subsection{Research Question}

The research question that guided the study is;

What is the influence of gender on the school administrative leadership scale (SALS). 


\subsection{Hypothesis}

The null hypothesis for the study was tested at an alpha level of 0.05 .

$\mathrm{Ho}_{1}$ : The influence of gender on the mean responses of principals on the school administrative leadership scale will not be statistically significant.

\section{Research Method}

The study is an instrumentation study. An instrumentation study is a type of research study that focuses on introduction of new or modified content, procedure, technologies or instruments of educational practice [4]. The study was carried out in Enugu State, Nigeria.

There are six education zones in Enugu State, Nigeria with two hundred and sixty two secondary schools. All the principals comprised the population as well as the sample since the population was not large. The instrument for the study is a 26 item developed school administrative leadership scale (SALS). The instrument was validated using factor analytic procedures (i.e. principal factor and normal varimax rotation). The 26 items that survived the validation exercise were also subjected to reliability assessment. It is a likert type scale scored on 4-point basis. Copies of the 26-item school administrative leadership scale (SALS) were administered to all the principals used for the study.

Research question 1 was answered using mean and standard deviation while the hypothesis was tested using t-test of difference between means of samples.

\section{Table 1. Summary of the Factor Loadings of the Surviving Twenty-Six Items of the SALS}

\begin{tabular}{|c|c|c|c|c|}
\hline \multicolumn{2}{|c|}{ Item } & \multirow[t]{2}{*}{ Item Structure } & \multirow[t]{2}{*}{ Loading } & \multirow[t]{2}{*}{ Comm. } \\
\hline $\begin{array}{l}\text { Dra } \\
\mathrm{ft}\end{array}$ & $\begin{array}{l}\text { Valid } \\
\text { ated }\end{array}$ & & & \\
\hline 1 & 1 & $\begin{array}{l}\text { Act without } \\
\text { consulting } \\
\text { your staff. }\end{array}$ & .72143 & .66982 \\
\hline 5 & 5 & $\begin{array}{l}\text { Refuse to } \\
\text { explain his } \\
\text { actions }\end{array}$ & .88165 & .80523 \\
\hline 7 & 7 & $\begin{array}{l}\text { Make all class } \\
\text { scheduling }\end{array}$ & .87389 & .83054 \\
\hline 11 & 11 & $\begin{array}{l}\text { Keep records } \\
\text { of defaulting } \\
\text { teachers } \\
\text { through class } \\
\text { records }\end{array}$ & .84467 & .80201 \\
\hline 13 & 13 & Delegate & .87662 & .81121 \\
\hline
\end{tabular}

\begin{tabular}{|c|c|c|c|c|}
\hline & & $\begin{array}{l}\text { responsibilitie } \\
\mathrm{s} \text { to deserving } \\
\text { staff }\end{array}$ & & \\
\hline 15 & 14 & $\begin{array}{l}\text { Treat all } \\
\text { members of } \\
\text { staff as } \\
\text { colleague }\end{array}$ & .86158 & .81091 \\
\hline 20 & 19 & $\begin{array}{l}\text { Use positive } \\
\text { reinforcement } \\
\text { to motivate } \\
\text { staff }\end{array}$ & .58291 & .42909 \\
\hline 25 & 21 & $\begin{array}{l}\text { Provide } \\
\text { incentives to } \\
\text { challenge } \\
\text { staff } \\
\text { productivity }\end{array}$ & .88903 & .83874 \\
\hline 30 & 24 & $\begin{array}{l}\text { Show interest } \\
\text { in negotiation } \\
\text { for better } \\
\text { condition for } \\
\text { teachers }\end{array}$ & .85040 & .82203 \\
\hline 2 & 2 & $\begin{array}{l}\text { Insist on } \\
\text { implementing } \\
\text { changes }\end{array}$ & .83939 & .75801 \\
\hline 3 & 3 & $\begin{array}{l}\text { Are known } \\
\text { for high } \\
\text { expectations } \\
\text { from staff }\end{array}$ & .84052 & .78428 \\
\hline 6 & 6 & $\begin{array}{l}\text { Insists on } \\
\text { standard rules } \\
\text { and } \\
\text { regulations }\end{array}$ & .90618 & .84592 \\
\hline 8 & 8 & $\begin{array}{l}\text { Dominate the } \\
\text { staff meeting }\end{array}$ & .88903 & .83874 \\
\hline 19 & 18 & $\begin{array}{l}\text { Let staff } \\
\text { members } \\
\text { know what is } \\
\text { expected of } \\
\text { them }\end{array}$ & .96700 & .99447 \\
\hline 29 & 23 & $\begin{array}{l}\text { Inform } \\
\text { teachers about } \\
\text { new ideas he } \\
\text { has come } \\
\text { across }\end{array}$ & .96700 & .99447 \\
\hline 31 & 25 & $\begin{array}{l}\text { Explain any } \\
\text { default and } \\
\text { apologizes }\end{array}$ & .96700 & .99447 \\
\hline 10 & 10 & $\begin{array}{l}\text { Resist late } \\
\text { coming of } \\
\text { staff }\end{array}$ & .87342 & .84569 \\
\hline 12 & 12 & $\begin{array}{l}\text { Keep tracks } \\
\text { of teachers } \\
\text { through class } \\
\text { records }\end{array}$ & .90427 & .80201 \\
\hline 16 & 15 & $\begin{array}{l}\text { Are friendly } \\
\text { and } \\
\text { approachable }\end{array}$ & .85040 & .81091 \\
\hline 17 & 16 & $\begin{array}{l}\text { Support } \\
\text { productivity } \\
\text { of teachers }\end{array}$ & .55040 & .82203 \\
\hline
\end{tabular}




\begin{tabular}{|c|c|c|c|c|}
\hline 4 & 4 & $\begin{array}{l}\text { Insist on } \\
\text { deadlines } \\
\text { being met }\end{array}$ & .90754 & .85875 \\
\hline 9 & 9 & $\begin{array}{l}\text { Programme } \\
\text { tight work for } \\
\text { teachers }\end{array}$ & .86201 & .80614 \\
\hline 18 & 17 & $\begin{array}{l}\text { Consider } \\
\text { teachers in } \\
\text { assigning } \\
\text { responsibilitie } \\
\text { s. }\end{array}$ & .85571 & .99447 \\
\hline 23 & 20 & $\begin{array}{l}\text { Have good } \\
\text { rapport with } \\
\text { staff }\end{array}$ & .90427 & .88661 \\
\hline 26 & 22 & $\begin{array}{l}\text { Provide } \\
\text { teachers with } \\
\text { suitable } \\
\text { materials for } \\
\text { teaching }\end{array}$ & .78818 & .72499 \\
\hline 35 & 26 & $\begin{array}{l}\text { Show interest } \\
\text { in team work } \\
\text { for the } \\
\text { general } \\
\text { welfare of the } \\
\text { school }\end{array}$ & .68988 & .55530 \\
\hline
\end{tabular}

\section{Results}

Results are presented in tables according to research questions and hypothesis.

\subsection{Research Question 1}

What is the influence of gender on the school administrative leadership scale?

Table 2. Mean Ratings of the Principals' Response on the SALS Based on Gender

\begin{tabular}{|l|l|l|l|l|l|}
\hline \multicolumn{2}{|c|}{ Items } & \multicolumn{2}{c|}{ Males } & \multicolumn{2}{c|}{ Females } \\
\hline Draft & $\begin{array}{l}\text { Vali } \\
\text { date } \\
\text { dat }\end{array}$ & Mean & SD & Mean & SD \\
\hline 1 & 1 & 2.8209 & .823 & 2.6393 & .932 \\
\hline 2 & 2 & 3.1294 & .814 & 2.6230 & 1.003 \\
\hline 3 & 3 & 2.9104 & .896 & 2.6230 & 1.019 \\
\hline 4 & 4 & 2.9701 & .748 & 2.6393 & 1.033 \\
\hline 5 & 5 & 3.0050 & .840 & 2.5738 & 1.024 \\
\hline 6 & 6 & 3.0348 & .751 & 2.5902 & 1.039 \\
\hline 7 & 7 & 3.0299 & .787 & 2.6066 & 1.021 \\
\hline 8 & 8 & 2.9453 & .826 & 2.6230 & 1.035 \\
\hline 9 & 9 & 3.0995 & .837 & 2.6721 & 1.060 \\
\hline 10 & 10 & 3.0796 & .868 & 2.7049 & 1.070 \\
\hline 11 & 11 & 3.0348 & .851 & 2.7213 & 1.051 \\
\hline 12 & 12 & 3.0995 & .787 & 2.7049 & 1.054 \\
\hline
\end{tabular}

\begin{tabular}{|l|l|l|l|l|l|}
\hline 13 & 13 & 3.0498 & .798 & 2.6557 & .998 \\
\hline 15 & 14 & 3.0498 & .899 & 2.6721 & 1.012 \\
\hline 16 & 15 & 2.9950 & .857 & 2.6885 & 1.073 \\
\hline 17 & 16 & 3.0199 & .836 & 2.6393 & 1.049 \\
\hline 18 & 17 & 3.1294 & .891 & 2.6066 & 1.037 \\
\hline 19 & 18 & 3.1493 & .269 & 2.5738 & 1.024 \\
\hline 20 & 19 & 3.0945 & .846 & 2.7377 & 1.471 \\
\hline 23 & 20 & 3.0995 & .787 & 2.7049 & 1.054 \\
\hline 25 & 21 & 2.9453 & .826 & 2.6230 & 1.035 \\
\hline 26 & 22 & 2.9303 & .897 & 2.7049 & 1.070 \\
\hline 29 & 23 & 3.1493 & 2.269 & 2.5738 & 1.024 \\
\hline 30 & 24 & 3.0199 & .836 & 2.6393 & 1.049 \\
\hline 31 & 25 & 3.1493 & 2.269 & 2.5738 & 1.024 \\
\hline 35 & 26 & 2.7662 & .849 & 3.3279 & .769 \\
\hline
\end{tabular}

Table 2 shows that for all the 26 items of the SALS there is a pronounced difference in the responses of males and females except in items 1, 19 and 22. This implies that the SALS is sensitive in detecting the leader behavior differences of male and female principals.

\subsection{Hypothesis}

Ho: The influence of gender on the mean responses of principals to the SALS will not be statistically significant.

This hypothesis was tested using the t-test of difference between means of independent samples.

Table 3. Shows Summary of t-test of Difference in the Mean Responses of Male and Female Principals on the SALS Df $=\mathbf{2 6 0}$

\begin{tabular}{|c|c|c|c|c|c|c|c|c|}
\hline $\begin{array}{l}\mathbf{e} \\
\mathbf{m}\end{array}$ & $\begin{array}{c}\text { Cat } \\
\text { egor } \\
\text { y of } \\
\text { Res } \\
\text { pon } \\
\text { dent } \\
\text { s }\end{array}$ & $\mathbf{N}$ & Mean & SD & t-cal & Alpha & t-cv & Decn. \\
\hline 1 & $\begin{array}{l}\mathrm{M} \\
\mathrm{F}\end{array}$ & $\begin{array}{l}201 \\
61\end{array}$ & $\begin{array}{l}2.82 \\
2.64\end{array}$ & $\begin{array}{l}.823 \\
.932\end{array}$ & 1.37 & 0.05 & 1.96 & $\begin{array}{l}\text { Uph- } \\
\text { old } \\
\text { HO }\end{array}$ \\
\hline 2 & $\begin{array}{l}\mathrm{M} \\
\mathrm{F}\end{array}$ & $\begin{array}{l}201 \\
61\end{array}$ & $\begin{array}{l}3.13 \\
2.62\end{array}$ & $\begin{array}{l}.814 \\
1.00 \\
3\end{array}$ & 3.60 & 0.05 & 1.96 & $\begin{array}{l}\text { Reject } \\
\text { HO }\end{array}$ \\
\hline 3 & $\begin{array}{l}\text { M } \\
F\end{array}$ & $\begin{array}{l}201 \\
61\end{array}$ & $\begin{array}{l}2.91 \\
2.62\end{array}$ & $\begin{array}{l}.896 \\
1.01 \\
9\end{array}$ & 1.98 & 0.05 & 1.96 & $\begin{array}{l}\text { Reject } \\
\text { HO }\end{array}$ \\
\hline 4 & $\begin{array}{l}\mathrm{M} \\
\mathrm{F}\end{array}$ & $\begin{array}{l}201 \\
61\end{array}$ & $\begin{array}{l}2.97 \\
2.64\end{array}$ & $\begin{array}{l}.748 \\
1.03 \\
3 \\
\end{array}$ & 2.32 & 0.05 & 1.96 & $\begin{array}{l}\text { Reject } \\
\text { HO }\end{array}$ \\
\hline 5 & $\begin{array}{l}\mathrm{M} \\
\mathrm{F}\end{array}$ & $\begin{array}{l}201 \\
61\end{array}$ & $\begin{array}{l}3.00 \\
2.57\end{array}$ & $\begin{array}{l}.840 \\
1.02 \\
4\end{array}$ & 3.00 & 0.05 & 1.96 & $\begin{array}{l}\text { Reject } \\
\text { HO }\end{array}$ \\
\hline 6 & $\begin{array}{l}\mathrm{M} \\
\mathrm{F}\end{array}$ & $\begin{array}{l}201 \\
61\end{array}$ & $\begin{array}{l}3.034 \\
8 \\
2.590 \\
2\end{array}$ & $\begin{array}{l}.757 \\
1.03 \\
9\end{array}$ & 3.10 & 0.05 & 1.96 & $\begin{array}{l}\text { Reject } \\
\text { HO }\end{array}$ \\
\hline 7 & $\begin{array}{l}\mathrm{M} \\
\mathrm{F}\end{array}$ & $\begin{array}{l}201 \\
61\end{array}$ & $\begin{array}{l}3.029 \\
9 \\
2.606 \\
6\end{array}$ & $\begin{array}{l}.787 \\
1.02 \\
1\end{array}$ & 2.98 & 0.05 & 1.96 & $\begin{array}{l}\text { Reject } \\
\text { HO }\end{array}$ \\
\hline 8 & $\begin{array}{l}\mathrm{M} \\
\mathrm{F}\end{array}$ & $\begin{array}{l}201 \\
61\end{array}$ & $\begin{array}{l}2.945 \\
3 \\
2.623\end{array}$ & $\begin{array}{l}.826 \\
1.03 \\
5\end{array}$ & 2.23 & 0.05 & 1.96 & $\begin{array}{l}\text { Reject } \\
\text { HO }\end{array}$ \\
\hline
\end{tabular}




\begin{tabular}{|c|c|c|c|c|c|c|c|c|}
\hline & & & 0 & & & & & \\
\hline 9 & $\begin{array}{l}\mathrm{M} \\
\mathrm{F}\end{array}$ & $\begin{array}{l}201 \\
61\end{array}$ & $\begin{array}{l}3.099 \\
5 \\
2.672 \\
1 \\
\end{array}$ & $\begin{array}{l}.837 \\
1.06 \\
0\end{array}$ & 2.89 & 0.05 & 1.96 & $\begin{array}{l}\text { Reject } \\
\text { HO }\end{array}$ \\
\hline $\begin{array}{l}1 \\
0\end{array}$ & $\begin{array}{l}\mathrm{M} \\
\mathrm{F}\end{array}$ & $\begin{array}{l}201 \\
61\end{array}$ & $\begin{array}{l}3.079 \\
6 \\
2.704 \\
9 \\
\end{array}$ & $\begin{array}{l}.868 \\
1.07 \\
0\end{array}$ & 2.50 & 0.05 & 1.96 & $\begin{array}{l}\text { Reject } \\
\mathrm{HO}\end{array}$ \\
\hline $\begin{array}{l}1 \\
1\end{array}$ & $\begin{array}{l}\mathrm{M} \\
\mathrm{F}\end{array}$ & $\begin{array}{l}201 \\
61\end{array}$ & $\begin{array}{l}3.034 \\
8 \\
2.721 \\
3 \\
\end{array}$ & $\begin{array}{l}.851 \\
1.05 \\
1\end{array}$ & 2.13 & 0.05 & 1.96 & $\begin{array}{l}\text { Reject } \\
\text { HO }\end{array}$ \\
\hline $\begin{array}{l}1 \\
2\end{array}$ & $\begin{array}{l}\mathrm{M} \\
\mathrm{F}\end{array}$ & $\begin{array}{l}201 \\
61\end{array}$ & $\begin{array}{l}3.099 \\
5 \\
2.704 \\
9 \\
\end{array}$ & $\begin{array}{l}.787 \\
1.05 \\
4\end{array}$ & 2.70 & 0.05 & 1.96 & $\begin{array}{l}\text { Reject } \\
\text { HO }\end{array}$ \\
\hline $\begin{array}{l}1 \\
3\end{array}$ & $\begin{array}{l}\mathrm{M} \\
\mathrm{F}\end{array}$ & $\begin{array}{l}201 \\
61\end{array}$ & $\begin{array}{l}3.049 \\
8 \\
2.655 \\
7 \\
\end{array}$ & $\begin{array}{l}.798 \\
.998\end{array}$ & 2.82 & 0.05 & 1.96 & $\begin{array}{l}\text { Reject } \\
\text { HO }\end{array}$ \\
\hline $\begin{array}{l}1 \\
4\end{array}$ & $\begin{array}{l}\mathrm{M} \\
\mathrm{F}\end{array}$ & $\begin{array}{l}201 \\
61\end{array}$ & $\begin{array}{l}3.049 \\
8 \\
2.672 \\
1 \\
\end{array}$ & $\begin{array}{l}.899 \\
1.01 \\
2\end{array}$ & 2.62 & 0.05 & 1.96 & $\begin{array}{l}\text { Reject } \\
\text { HO }\end{array}$ \\
\hline $\begin{array}{l}1 \\
5\end{array}$ & $\begin{array}{l}\mathrm{M} \\
\mathrm{F}\end{array}$ & $\begin{array}{l}201 \\
61\end{array}$ & $\begin{array}{l}2.995 \\
0 \\
2.688 \\
5 \\
\end{array}$ & $\begin{array}{l}.857 \\
1.07 \\
3\end{array}$ & 2.04 & 0.05 & 1.96 & $\begin{array}{l}\text { Reject } \\
\mathrm{HO}\end{array}$ \\
\hline $\begin{array}{l}1 \\
6\end{array}$ & $\begin{array}{l}\mathrm{M} \\
\mathrm{F}\end{array}$ & $\begin{array}{l}201 \\
61\end{array}$ & $\begin{array}{l}3.019 \\
9 \\
2.639 \\
3 \\
\end{array}$ & $\begin{array}{l}.836 \\
1.04 \\
9\end{array}$ & 2.59 & 0.05 & 1.96 & $\begin{array}{l}\text { Reject } \\
\text { HO }\end{array}$ \\
\hline $\begin{array}{l}1 \\
7\end{array}$ & $\begin{array}{l}\mathrm{M} \\
\mathrm{F}\end{array}$ & $\begin{array}{l}201 \\
61\end{array}$ & $\begin{array}{l}3.129 \\
4 \\
2.606 \\
6 \\
\end{array}$ & $\begin{array}{l}.891 \\
1.03 \\
7\end{array}$ & 3.56 & 0.05 & 1.96 & $\begin{array}{l}\text { Reject } \\
\text { HO }\end{array}$ \\
\hline $\begin{array}{l}1 \\
8\end{array}$ & $\begin{array}{l}\mathrm{M} \\
\mathrm{F}\end{array}$ & $\begin{array}{l}201 \\
61\end{array}$ & $\begin{array}{l}3.149 \\
3 \\
2.573 \\
8 \\
\end{array}$ & $\begin{array}{l}2.26 \\
9 \\
1.02 \\
4 \\
\end{array}$ & 2.78 & 0.05 & 1.96 & $\begin{array}{l}\text { Reject } \\
\text { HO }\end{array}$ \\
\hline $\begin{array}{l}1 \\
9\end{array}$ & $\begin{array}{l}\mathrm{M} \\
\mathrm{F}\end{array}$ & $\begin{array}{l}201 \\
61\end{array}$ & $\begin{array}{l}3.094 \\
5 \\
2.737 \\
7 \\
\end{array}$ & $\begin{array}{l}.846 \\
1.47 \\
1\end{array}$ & 1.81 & 0.05 & 1.96 & $\begin{array}{l}\text { Uph- } \\
\text { old } \\
\text { HO }\end{array}$ \\
\hline $\begin{array}{l}2 \\
0\end{array}$ & $\begin{array}{l}\mathrm{M} \\
\mathrm{F}\end{array}$ & $\begin{array}{l}201 \\
61\end{array}$ & $\begin{array}{l}3.099 \\
5 \\
2.704 \\
9 \\
\end{array}$ & $\begin{array}{l}.787 \\
1.05 \\
4\end{array}$ & 2.70 & 0.05 & 1.96 & $\begin{array}{l}\text { Reject } \\
\mathrm{HO}\end{array}$ \\
\hline $\begin{array}{l}2 \\
1\end{array}$ & $\begin{array}{l}\mathrm{M} \\
\mathrm{F}\end{array}$ & $\begin{array}{l}201 \\
61\end{array}$ & $\begin{array}{l}2.945 \\
3 \\
2.623 \\
0 \\
\end{array}$ & $\begin{array}{l}.826 \\
1.03 \\
5\end{array}$ & 2.23 & 0.05 & 1.96 & $\begin{array}{l}\text { Reject } \\
\text { HO }\end{array}$ \\
\hline $\begin{array}{l}2 \\
2\end{array}$ & $\begin{array}{l}\mathrm{M} \\
\mathrm{F}\end{array}$ & $\begin{array}{l}201 \\
61\end{array}$ & $\begin{array}{l}2.930 \\
3 \\
2.704 \\
9 \\
\end{array}$ & $\begin{array}{l}.897 \\
1.07 \\
0\end{array}$ & 1.49 & 0.05 & 1.96 & $\begin{array}{l}\text { Uph- } \\
\text { old } \\
\text { HO }\end{array}$ \\
\hline $\begin{array}{l}2 \\
3\end{array}$ & $\begin{array}{l}\mathrm{M} \\
\mathrm{F}\end{array}$ & $\begin{array}{l}201 \\
61\end{array}$ & $\begin{array}{l}3.149 \\
3 \\
2.573 \\
8 \\
\end{array}$ & $\begin{array}{l}2.26 \\
9 \\
1.02 \\
4 \\
\end{array}$ & 2.78 & 0.05 & 1.96 & $\begin{array}{l}\text { Reject } \\
\text { HO }\end{array}$ \\
\hline $\begin{array}{l}2 \\
4\end{array}$ & $\begin{array}{l}\mathrm{M} \\
\mathrm{F}\end{array}$ & $\begin{array}{l}201 \\
61\end{array}$ & $\begin{array}{l}3.019 \\
9 \\
2.639 \\
3 \\
\end{array}$ & $\begin{array}{l}.836 \\
1.04 \\
9\end{array}$ & 2.59 & 0.05 & 1.96 & $\begin{array}{l}\text { Reject } \\
\text { HO }\end{array}$ \\
\hline $\begin{array}{l}2 \\
5\end{array}$ & $\begin{array}{l}\mathrm{M} \\
\mathrm{F}\end{array}$ & $\begin{array}{l}201 \\
61\end{array}$ & $\begin{array}{l}3.149 \\
3 \\
2.573 \\
8 \\
\end{array}$ & $\begin{array}{l}2.26 \\
9 \\
1.02 \\
4 \\
\end{array}$ & 2.78 & 0.05 & 1.96 & $\begin{array}{l}\text { Reject } \\
\text { HO }\end{array}$ \\
\hline $\begin{array}{l}2 \\
6\end{array}$ & $\begin{array}{l}\mathrm{M} \\
\mathrm{F}\end{array}$ & $\begin{array}{l}201 \\
61\end{array}$ & $\begin{array}{l}2.766 \\
2 \\
3.327 \\
9 \\
\end{array}$ & $\begin{array}{l}.849 \\
.769\end{array}$ & 4.88 & 0.05 & 1.96 & $\begin{array}{l}\text { Reject } \\
\text { HO }\end{array}$ \\
\hline
\end{tabular}

Summary of result in table 3 reveals that for items 2 , $3,4,5,6,7,8,9,10,11,12,13,14,15,16,17,18,20$, $21,23,24,25$ and 26 of the SALS, the calculated values were greater than the critical value at an alpha level of 0.05 . For these items the null hypothesis were rejected indicating a significant difference in mean responses of male and female principals on those items. For items 1, 19 and 22, the calculated t-values were less than the critical t-values of 1.96 at the alpha level of 0.05 . For these three items, the null hypothesis was upheld indicating a significant convergence in the leader behavior of male and female principals in those aspects of leadership skills.

\section{Discussion of Findings}

The extent to which the instrument is sensitive to gender differences in leadership skills of the principals was assessed by determining the mean differences in the responses of males and females on the instrument. Summary of result in table 2 reveals that for all the twenty six items of the SALS, there is a pronounced difference in the responses of males and females except in items 1, 19, and 22. This implies that the SALS is sensitive in detecting the leader behavior differences of male and female principals.

This finding is consistent with current theories of leadership as it pertains to gender determinants of leadership structures [6] [7] [9] [10]. Moorhead and Griffin noted that in terms of leadership, females are more authoritarian than males [7]. This suggests that within the secondary school system, a marginal difference is expected in the leader behavior of male and female principals. Vroom in his famous theories of leadership also provided a model that depicts pronounced differences in leader skills of individuals arising from gender differences [10]. He went ahead to trace the differences in leader behavior of males and females to hormonal effects. In his famous essay, he stresses that hormonal reactions, which produces marginal differences in dispositional behaviors of male and females are different. While it triggers off the temperament of females, it induces more of consideration behavior in males. Meluiam presented his own argument from a psychological perspective [6]. Rather than believing that the differences in leader behavior of males and females, he argued that the authoritarian display of female leaders is a function of complex.

\section{Conclusion}

Based on the result obtained in this instrumentation research, the researcher concludes that the developed school administrative leadership scale (SALS) is very sensitive to gender differences in the measurement of leadership skills of school principals.

\section{Recommendations}

Based on the findings of this study, the researcher recommends that: 
1. Gender differences should be taken into consideration in the assessment and appointment of school principals.

2. Since a valid and stable instrument has been developed, in the leadership domain, measurement of principals leadership skills should constitute a regular practice by both the education ministries and post primary schools management board.

3. Researchers in the field of leadership should also use this instrument in the course of their research.

\section{References}

[1] Conger, J.A. (1989). The charismatic leader: Behind the mystique of exceptional leadership. San Francisco. JosseyBass http://books.google.com.ng. Retrieved 20/6/14.

[2] Elechi, C.N. (2013). Development and validation of leadership skill assessment scale for secondary school principals. Journal of Educational and Social research 3 (5) 9-16

[3] en.wikipedia.org/wiki/leadership Retrieved 20/6/14.

[4] ICEE (1982). Priorities in Educational Research. Washington: Test Development Unit.

[5] Mcshane, S.L. and VonGlinow, M.A. (2010). Organizational

behaviour. Boston. McGraw Hills.

[6] Meluium, H. (1980). Some correlates of authoritarianism in two cultural groups. Journal of psychology, 73, 27 -

32.

[7] Moorhead, A and Griffin (2004). Organizational behaviour: Managing people and organizations. New York: Houghton Miffin Company.

[8] Post Primary Schools Management Board (PPSMB)

(2004). Guide on promotion/appointment of principals. Enugu: PPSMB vol 1 No. 253.

[9] Shelly, A.K. and Locke, E.A. (1991). Leadership. Do skills matter? Handbook of principles of organizational behaviour. Academy of management executive 3(1) $48-60$.

[10] Vroom, V.H. (1984). Leader authoritarianism and employee attitudes. Personal psychology 13 (2) 125-140.

[11] Vroom, V.H. and Jago A.G. (1993). The new leadership. New Jersey. Prentice Hall.

[12] Wiki.answers.com. What is demographic factors. Retrieved 20/6/2014.
[13] www.businessdictionary.com What are demographic factors. Retrieved 20/6/14.

[14] www.siyli.org/what is leadership. Retrieved 22/6/2014. 